\title{
Research on Innovation Risk Management based on Bayesian Risk Decision-Making
}

\author{
Yingchun Guo \\ College of Mathematics \& Computer Seience \\ Hebei University \\ Baoding 071002, China
}

Tel: +86-132-929-982-10Ｅ-mail: guoyc@hbu.cn

Received: November 24, 2011

Accepted: December 16, 2011 Published: January 5, 2012

doi:10.5430/ijba.v3n1p21

URL: http://dx.doi.org/10.5430/ijba.v3n1p21

This paper is a part of the project "Research on Innovation Risk Management Mechanism of Industrial Chain" of Hebei Soft Science Program.

\begin{abstract}
Innovation is an inexhaustible force for the prosperity of one nation, and also the life source of enterprises. However, the high-risk characteristics of innovation activities make enterprises to perform scientific and effective innovation risk management. Based on a general introduction of Bayestian Risk Decision-making Theory and practices of product innovation in enterprises, the author discusses how to use the theory to achieve quantitative innovation-risk management, providing references for scientific decision of innovation activities in enterprises.
\end{abstract}

Keywords: Innovation Risk, Risk Management, Bayesian Risk Decision-Making

\section{Introduction}

Innovation is the soul of a nation's progress, an inexhaustible force for the prosperity of a nation, and the life source of enterprises (Siwei Cheng, 2009, p1-14). Without innovations, enterprises would not be able to upgrade the production structure. With weakening competitiveness, enterprises will die. However, innovation is a "double-edged sword", with characteristics of high potentials, high inputs, high returns, and high risks. Particularly, high risks from technologies, market, and management frustrate or even kill many innovation activities, which may even threaten the healthy development of human society. Therefore, to manage the innovation risks is significant.

Currently, most researches on innovations are about methods and modes that promote enterprises to develop independent innovations, seldom focus on innovation risks. Xiaofang and Jianjun Hao (2010) built an application framework for high-tech enterprises implementing overall risk management, according to characteristics of risks in front of high-tech enterprises. Mei Zhao, Hongzhi Yue, and Yan Yang (2007) studied the whole process of continuous risk management of high-tech enterprises. Rong Liu and Keyi Wang (2009) proposed a synthesized risk management mode for enterprises' coorperative innovations based on the meta-synthesis method. Yujun Miao (2010), Xiaofeng Li, and Jiuping Xu (2010) put forward the risk management strategy in the process of technological innovation, which could help to achieve effective risk prevention. Zhaoyang Pan and Yunzhi Liang (2009) studied the risk management of technological innovation in perspective of venture capital. All these literatures were qualitative studies on different stages of risk management, including risk planning, risk identification, risk analysis, and risk process.

In the theoretical field, there are quantitative researches on innovation risk management. Zhe Song, Shu'en Wang, and Zhou Liu (2010) proposed the synthesis evaluation method based on the Analytic Network Process and the Grey Relational Analysis and applied it to the risk evaluation of enterprises' technological innovation. Junwen Xing, Baoshan Chi and Feng Liu (2008) put forward a system structure, grades and standards, and quantitative method for the quantitative indexes of technological risk event, and built a three-parameter quantitative model for technological risk. Yang Chen and Yuejin Tan (2007) studied the risk estimation methods for personalized product innovation projects from the characteristics of products' market life cycle. Xiaofeng Li, Jiuping Xu and Jinjiang Yan (2010) built a risk pre-warning system for enterprises' technological innovation projects. Zhengyuan Jia and Liang Zhao (2009) built a comprehensive evaluation mode for multi-objective decisions based on the probability distribution evaluation theory of 
intervals, and made a comprehensive evaluation of venture capital. Andrew Kusiak (2009) proposed a production innovation program driven by market or customer data. These researches promoted the scientific decision of technological risk management, but the application is unsatisfying. On one hand, these methods are too complicated to use in enterprises. On the other hand, most quantitative studies focus on the risk evaluation, but seldom on risk decision.

The risk decision-making is to make decision according to incomplete information. According to the objective of risk management, with basis of risk identification and risk evaluation, make reasonable choice and combination of different risk management methods, and offer a specific program for risk management. Faced high risks from technologies, market, and management, enterprise managers should master the scientific and feasible risk decision-making method, managing innovation risks effectively. This paper is to explore the effective quantitative risk decision-making method, in order to help enterprise managers to achieve effective innovation risk management.

Bayesian approach is a powerful tool for risk decision-making (Richard Bradley, 2007; Xiaomo Jiang \& Sankaran Mahadevan, 2007). Due to its convenience and easiness, this approach is applying in many fields. Jacobus P. Venter and Cornelis C. V. Waveren (2009) used the Bayesian Decision technology to support the new product development management. Rajkumar Venkatesan, V. Kumar, and Timothy Bohling (2007) applied the Bayesian Risk Decision-Making Theory to the choice of customers in customer relationship management. Kwai-Sang Chin, Da-wei Tang, and Jian-bo Yang (2009) applied the Bayesian network method to the risk evaluation in new product R \& D. Paul L. Reynolds and Geoff Lancaster (2007) proposed a Bayesian solution for enterprises predicting the strategic marketing management decision. Min Chen, Yusen Xia, and Xinlei Wang (2010) built a Bayesian model to achieve dynamic knowledge update, in order to deal with the supply uncertainties and risks.

\section{An Introduction of Bayesian Risk Decision-Making Theory}

\subsection{Theory}

Risk decision-making decision runs through the whole risk management process. By analyzing risks and losses scientifically, it can help to choose the reasonable risk management techniques and methods and finally get the most satisfying solution from several options. Every risk decision-making includes three elements: the state group consisted of different natural status, the action group consisted of a set of actions taken by decision makers, and the description of utility or losses from different combinations of statues and actions. From the three elements, we can get different risk conditions. Once the decision maker makes a decision with uncertain result, it means certain risk. The risk decision-making needs to get changeable market information by increasing inputs. Based on mastering various natural conditions in time, use the collected information reasonably, and select the decision scientifically, reducing risks, and improving economic and social benefits. In risk decision-making, the accuracy of estimation of natural conditions can directly affect the expected returns. In order to make better decision, it needs to update the information in time. After getting new information, we can revise the original estimated probability of emergence of certain natural condition, and use the revised probability distribution to make new decision. Because the probability correction is based on the Bayesian Theorem in probability theory, this decision is called Bayesian Decision.

\subsection{Procedures}

Bayesian Risk Decision-Making has three steps:

\subsubsection{Prior Analysis}

First, evaluate the probability ${ }_{P\left(N_{i}\right)}(i=1,2, \cdots, m)$ of emergence of natural state $N_{i} \cdot u_{i j}$ is the utility of program $d_{j}(j=1,2, \cdots, n)$ under the status $N_{i}$. See Table 1 . According to the law of expectation, calculate the expected utility of each program: $E\left(d_{j}\right)=\sum_{i=1}^{m} P\left(N_{i}\right) u_{i j}$, $(j=1, \ldots, n)$. Accordingly, the optimal solution and expected utility is ${ }_{\max E\left(d_{j}\right)=E\left(d_{k}\right)=E M U}$.

\subsubsection{Prediction Posterior Analysis}

In prediction posterior analysis, estimate the value of complete information first and take it as a standard. If the cost for supplementing information is far less than the value of complete information, the supplementation will be economical.

Otherwise, if the cost is close to or even higher than the value of complete information, the supplementation will be 
uneconomical. As the prediction of complete information is in the state $N_{k}$, it becomes a decision-making under certainty. The optimal program should be established by the formula $\max _{j}\left\{u_{k j}\right\}$. Then, with complete information, the maximum expected utility from the decision should be $E U P I=\sum_{k=1}^{m} P\left(N_{k}\right) \underset{1 \leq j \leq n}{\max }\left\{u_{k j}\right\}$.

Apparently, the balance between EUPI and EMU is the increasing part of expected utility due to the complete information. It is the expected value of complete information, EVPI for short, and EVPI $=E U P I-E M U$. EVPI, as the top for the cost of information, determine whether it is necessary to obtain more information or not.

\subsubsection{Posterior Analysis}

Posterior analysis includes supplementing new information, calculating and revising probability, posterior decision, and calculating the value of supplement information.

1. Supplement new information. Investigate, explore, and consult the $S$ statues of $X_{1}, X_{2}, \ldots, X_{s}$ and predict which one will appear. Meanwhile, get the conditional probability ${ }_{P\left(X_{j} \mid N_{i}\right)}$ by materials, namely the probability of prediction $X_{j}$ as the natural state $N_{i}$ really appears.

2. Revise the probability. Based on the prior probability $P\left(N_{i}\right)(i=1,2, \cdots, m)$ and the conditional probability $P\left(X_{j} \mid N_{i}\right)(i=1,2, \cdots, m ; j=1,2, \cdots, s)$, calculate the probability distribution of $X_{j}: P\left(X_{j}\right)=\sum_{i=1}^{m} P\left(N_{i}\right) P\left(X_{j} \mid N_{i}\right)$. Use the Bayesian formula to calculate the revised probability and get the posterior probability $P\left(N_{i} \mid X_{j}\right)=\frac{P\left(N_{i}\right) P\left(X_{j} \mid N_{i}\right)}{P\left(X_{j}\right)}$.

3. Posterior decision. According to the posterior probability, we can make the decision-making framework. Suppose the supplement information predict that the state $X_{k}$ appears, use the posterior revised probability distribution $P\left(N_{i} \mid X_{k}\right)(i=1,2, \ldots, m)$ to calculate the expected utility, which is the basis for decision- making. $E\left(d_{j} \mid X_{k}\right)=\sum_{i=1}^{m} P\left(N_{i} \mid X_{k}\right) u_{i j}, \quad(j=1,2, \cdots, n ; \quad k=1, \quad 2, \quad \ldots, \quad S \quad) . \quad$ The maximum expected utility is $E\left(X_{k}\right)=\max _{j} E\left(d_{j} \mid X_{k}\right)={ }_{E}\left(d_{j k} \mid X_{k}\right)$. Once get the prediction from supplement information, we can choose the optimal program with maximum expected utility $d_{j k}$ in prediction $X_{k}$, and make the decision.

4. Calculate the value of supplement information. Use the supplement information to predict the probability of emergence of each state $P\left(X_{i}\right)(i=1,2, \ldots, S)$ and calculate the maximum expected utility in posterior 
analysis $E M U^{*}=\sum_{i=1}^{m} P\left(X_{i}\right) E\left(X_{i}\right)$. Apparently, after getting the supplement information, the expected utility rises by $E M U^{*}-E M U$. Therefore, the value of supplement information is $E M U^{*}-E M U$. Then, compare the value of supplement information with the cost for getting information and make the right decision. Because the supplement information is usually uncertainty, this information is incomplete or not accurate. It is also known as the sample information.

\section{Innovation Risk Management Cases}

\subsection{Three Elements for Innovation Risk Management Decision}

\subsubsection{The Group of Natural States}

The comprehensive evaluation on innovation activity is $N=\left\{N_{1}, N_{2}, \cdots, N_{m}\right\}$. For instance, $\mathrm{N}_{1}$ stands for best, $\mathrm{N}_{2}$ stands for better, $\ldots$, and $\mathrm{N}_{\mathrm{m}}$ stands for worst. Experts give the prediction posterior probability of each state $P\left(N_{i}\right),(i=1, \ldots, m)$.

\subsubsection{The Group of Actions}

The action toward innovation activity is $D=\left\{d_{1}, d_{2}, \cdots, d_{n}\right\}$. Here $\mathrm{d} 1$ stands for high investment, such as more investment in $\mathrm{R} \& \mathrm{D}$, new production equipment, and new product. $\mathrm{d} 2$ stands for medium investment, such as medium investment in R \& D, and changes of product functions. d3 stands for low investment, such as changes of production techniques, and better product quality. $\mathrm{d} 4$ stands for no investment in innovation, such as only changes in packages or more advertisements.

\subsubsection{The Group of Descriptions of Utility or Losses}

$U=\left(u_{i j}\right)_{m n}$. Here, $u_{i j} \in[-100,100]$ is the economic utility that can be evaluated by money, or the utility function evaluated by non-monetary factors. Here, we suggest the second meaning, because innovation activities can not only generate economic benefits , but also social benefits, so as to bring intangible assets and long-term interests for enterprises. Here, the utility function can be measured by the satisfaction degree, such as enterprises' satisfaction degree, customers' satisfaction degree, expert scoring, and other comprehensive scores.

\subsection{Description of Product Innovation risk}

Suppose an enterprise starts a new product R \& D. There are five states of comprehensive evaluations on economic utility and social benefits $N=\left\{N_{1}, N_{2}, N_{3}, N_{4}, N_{5},\right\}$. Here, N1 stands for best, N2 stands for better, N3 stands for medium, N4 stands for worse, and N5 stands for worst. According to the data analysis of the market survey and the expert 
prediction, the probability distribution of each state is $P\left(N_{1}\right)=0.2, P\left(N_{2}\right)=0.4, P\left(N_{3}\right)=0.2, P\left(N_{4}\right)=0.15, P\left(N_{5}\right)=0.05$. The enterprise has four options $D=\left\{d_{1}, d_{2}, d_{3}, d_{4}\right\}$. d1 stands for high investment, d2 stands for medium investment, d3 stands for low investment, and d4 stands for no investment. The utility of four options under different states is in Table 2.

Data description: the expected utility declines along with the diminishing prospect of market state. For instance:

$u_{11}$ : under the high investment and best market conditions, the economic utility and social benefits reach the highest. The expected utility $u_{11}=100 ; u_{21}$ : under the high investment and better market conditions, the economic utility and social benefits are high. The expected utility $u_{21}=70 ; u_{31}$ : under the high investment and ordinary market conditions, the economic utility and social benefits are medium. The expected utility is $u_{31}=50$; $u_{41}$ : under the high investment and worse market conditions, the economic utility and social benefits are worse. The expected utility is $u_{41}=-20$. $u_{51}:$ under the high investment and worst market conditions, the enterprise suffers from serious losses. The expected utility

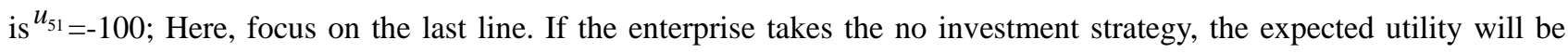
negative. For instance:

$u_{14}$ : the enterprise does not invest, though the market conditions are good. It will make the enterprise lose potential economic utility and social benefits. The expected utility $u_{14}=-80 ; u_{54}$ : the enterprise does not make innovation investment and the market conditions are bad. Then, there is no economic benefit or social benefit. The expected utility $u_{54}=0$.

\subsection{The Bayesian Risk Decision-Making Process}

\subsubsection{Prior Analysis}

According to the probability of natural state and the expected utility (see to Table 2), by following the law of expectation, calculate the expected utility of each program. $E\left(d_{j}\right)=\sum_{i=1}^{5} P\left(N_{i}\right) u_{i j}, j=1, \ldots, 4$. Accordingly, the optimal expectation for the optimal program is $\max _{j} E\left(d_{j}\right)=E\left(d_{k}\right)=E M U$. For instance, $E\left(d_{1}\right)=0.2 * 100+0.4 * 70+0.2 * 50+0.15 *(-20)+0.05 *(-100)=50$; similarly, $E\left(d_{2}\right)=55.5, E\left(d_{3}\right)=58.5, E\left(d_{4}\right)=-51$. Then, the optimal decision and the optimal expected utility is $E M U=E\left(d_{3}\right)=58.5$. It means that the enterprise can take the low-investment strategy if only with the prior information. 


\subsubsection{Prediction Posterior Analysis}

In prediction posterior analysis, estimate the value of complete information firstly. As the prediction of complete information is in the state ${ }^{N_{k}}$, it becomes the decision-making under certainty. Apparently, the optimal program is $\max _{j}\left\{u_{k j}\right\}$.

Then, with complete information, the maximum expected utility from decision-making is:

EUPI $=\sum_{k=1}^{5} P\left(N_{k}\right) \max _{1 \leq j \leq 4}\left\{u_{k j}\right\}=0.2 * 100+0.4 * 80+0.2 * 80+0.15 * 30+0.05 * 0=72.5$. Therefore, the value of complete information $E V P I=E U P I-E M U=72.5-58.5=14$. It means the value of complete information is equal to 14 units of utility.

\subsubsection{Posterior Analysis}

\section{Supplement new information}

According to the market conditions, investigate, explore, and consult the five states $X_{1}$ (excellent), $X_{2}$ (better), $X_{3}$ (medium), ${ }^{X_{4}}$ (worse), and $X_{5}$ (worst), and predict which one will appear. Meanwhile, get the conditional probability $P\left(X_{j} \mid N_{i}\right)$, which is the probability of predicting the emergence of ${ }^{X_{j}}$ when the natural state ${ }^{N_{i}}$ actually appears. (See Table 3)

\section{Revise the Probability}

Based on the prior probability $P\left(N_{i}\right)(i=1,2, \ldots, 5)$ and the conditional probability $P\left(X_{j} \mid N_{i}\right)(i=1,2, \ldots, 5 ; j=1,2, \ldots$, 5), calculate the probability distribution of $X_{j}$ :

$$
P\left(X_{j}\right)=\sum_{i=1}^{5} P\left(N_{i}\right) P\left(X_{j} \mid N_{i}\right) .
$$

For instance, $P\left(X_{1}\right)=0.2 * 0.5+0.4 * 0.2+0.2 * 0.1+0.15 * 0.05+0.05 * 0.05=0.21$. Similarly, $P\left(X_{2}\right)=0.3075, \quad P\left(X_{3}\right)=0.2475$, $P\left(X_{4}\right)=0.155$, and $P\left(X_{5}\right)=0.08$. Use the Bayesian formula to calculate the revised probability of ${ }^{N_{i}}$, namely the posterior probability (see to Table 4):

$$
P\left(N_{i} \mid X_{j}\right)=\frac{P\left(N_{i}\right) P\left(X_{j} \mid N_{i}\right)}{P\left(X_{j}\right)},\left(i_{=1}, 2, \ldots, 5 ; j=1,2, \ldots, 5\right) .
$$

\section{Posterior Decision}

Suppose the supplement information predicts the appearance of state ${ }^{X_{k}}$. Use the posterior revised probability 
distribution $P\left(N_{i} \mid X_{k}\right)(i=1,2, \ldots, 5)$ to calculate the expected utility of each program. By following the law of expectation, make the decision. Then, $E\left(d_{j} \mid X_{k}\right)=\sum_{i=1}^{5} P\left(N_{i} \mid X_{k}\right) u_{i j},(\mathrm{j}=1,2, \ldots, 5, \mathrm{k}=1,2, \ldots, 5)$.

For instance, if the market survey shows that the market condition is ${ }_{1}$, calculate the expected utility of $d_{k}$ (see to Table 5).

$E\left(d_{1} \mid X_{1}\right)=0.4762 * 100+0.381 * 70+0.0952 * 50+0.0357 *(-20)+0.0119 *(-100)=77.14$. Similarly, there is

$E\left(d_{2} \mid X_{1}\right)=68.93, \quad E\left(d_{3} \mid X_{1}\right)=63.45, \quad E\left(d_{4} \mid X_{1}\right)=-65.48$

Here, as the market condition is better, the enterprise can take the stategy ${ }^{d_{1}}$. The maximum expected utility is $E\left(d_{1} \mid X_{1}\right)=77.14$.

As the market condition is $X_{2}$, calculate and compare the expected utility of each $d_{k}$. The maximum expected utility is $E\left(d_{2} \mid X_{2}\right)=68.37$.

As the market condition is $X_{3}$, calculate and compare the expected utility of each $d_{k}$. The maximum expected utility is $E\left(d_{3} \mid X_{3}\right)=64.65$

As the market condition is $X_{4}$, calculate and compare the expected utility of each $d_{k}$. The maximum expected utility is $E\left(d_{3} \mid X_{4}\right)=44.49$

As the market condition is $X_{5}$, calculate and compare the expected utility of each $d_{k}$. The maximum expected utility is $E\left(d_{3} \mid X_{5}\right)=28.13$

4. Calculate the Value of Supplement Information

According to the calculated supplement information, predict the probability of each status $P\left(X_{i}\right)(i=1,2, \ldots, 5)$. Calculate the maximum expected utility in posterior analysis:

$E M U^{*}=\sum_{i=1}^{5} P\left(X_{i}\right) E\left(X_{i}\right)=0.21 * 77.14+0.3075 * 68.37+0.2475 * 64.65+0.155 * 44.19+0.08 * 28.13=62.325$

Apparently, after getting the supplement information, the expected utility rises: 
$E M U^{*}-E M U=62.325-58.5=3.825$. The value of supplement information is 3.825 unit of utility. Then, compare the value of supplement information and the cost for acquiring the information, and make the right decision.

\section{Conclusion}

The innovation risk management is critical for the survival and the development of enterprise. In this paper, taking the product innovation activity for instance, the author discusses the innovation risk management based on Bayesian Risk Decision-Making. Here, one point should be noted particularly: the repetitive application of Bayesian Risk Decision-Making can help the enterprise to carry out the dynamic risk management of innovation activities and adapt to the changing market conditions, achieving the scientific management of innovation risks.

\section{References}

Andrew, K. (2009). Innovation: a data-driven approach. International Journal of Production Economics. No.122(1). p440-448.

Chen, Y. \& Tan, Y. (2007). A method of product innovation risk estimation based on product market life cycle. Systems Engineering. No.25(5). p112-115.

Cheng, S. (2009). On the construction of an innovative country. China Soft Science. No.12. p1-14.

Jacobus, P. V., Cornelis, C. V. W. (2009). New product development with dynamic decision support. International Journal of Innovation and Technology Management. No.6(2). p155-167.

Jia, Z. \& Zhao, L. (2009). Venture capital management evaluation by probability distribution of interval number. Technoeconomics and Management Research. No.6. p7-10.

Kwai-Sang, C., Da-Wei, T. \& Jian-Bo, Y., etc. (2009). Assessing new product development project risk by Bayesian network with a systematic probability generation methodology. Expert Systems with Applications. No.36(6). p9879-9890.

Li, X. \& Xu, J. (2010). Study on risk decision of enterprises' technical innovation projects. Soft Science. No.24(10). p72-75.

Li, X., Xu, J. \& Yan, J. (2010). Study on the construction of risk early warning system for enterprise technological innovation projects. Journal of Sichuan University (Social Science Edition). No.5. p88-95.

Liu, R. \& Wang, K. (2009). Research on integrated management for enterprise's cooperation innovation. Science and Technology Management Research. No.10. p339-340.

Miao, Y. (2010). Enterprises in the process of technological innovation risks and prevention strategy. Science and Technology Management Research. No.7. p1-3.

Min, C., Yusen, X., Xinlei, W. (2010). Managing supply uncertainties through Bayesian information update. IEEE Transactions on Automation Science and Engineering. No.1. p24-36.

Pan, Z. \& Liang, Y. (2009). Technical innovation risk management in perspective of venture capital. Science \& Technology Progress and Policy. No.26(20). p13-16.

Paul, L. \& Reynolds, G. L. (2007). Predictive strategic marketing management decisions in small firms: A possible Bayesian solution. Management Decision. No.45(6). p1038-1057. http://dx.doi.org/10.1108/00251740710762062

Rajkumar, V. V. \& Kumar, T. B. (2007). Optimal customer relationship management using Bayesian Decision Theory: an application for customer selection. Journal of Marketing Research. No.XLIV(4). p579-597.

Richard, B. (2007). A unified Bayesian Decision Theory. Theory and Decision. No.63(3). p233-263. http://dx.doi.org/10.1007/s11238-007-9029-3

Song, Z. Wang, S. \& Liu, Z. (2010). Application of ANP-GRAP integrating method for risk evaluation in enterprises' technological innovation. Science of Science and Management of S. \& T. No.1. p55-58.

Xiao, F \& Hao, J. (2010). On the ERM implementation framework of high-tech enterprises. Scientific Management Research. No.28(2). p66-69.

Xiaomo, J. \& Sankaran, M. (2007). bayesian risk decision-making method for model validation under uncertainty. Reliability Engineering \& System Safety. No.92(6). p707-718.

Xing, J., Chi, B. \& Liu, F. (2008). Research on the three-parameter technical risk quantification model for R\&D project. System Engineering --- Theory \& Practice. No.10. p131-137.

Zhao, M. Yue, H. \& Yang, Y. (2007). Continuous risk management for high-tech enterprise technological innovation. Commercial Research. No.8. p33-39. 
Table 1. Utility

\begin{tabular}{|c|c|c|c|c|}
\hline State\&probability & $d_{1}$ & $d_{2}$ & $\cdots$ & $d_{n}$ \\
\cline { 1 - 2 }$N_{1} P\left(N_{1}\right)$ & $u_{11}$ & $u_{12}$ & $\cdots$ & $u_{1 n}$ \\
\hline$N_{2}: P\left(N_{2}\right)$ & $u_{21}$ & $u_{22}$ & $\cdots$ & $u_{2 n}$ \\
\hline$\ldots$ & $\cdots$ & $\cdots$ & $\cdots$ & $\cdots$ \\
\hline$N_{m}: P\left(N_{m}\right)$ & $u_{m 1}$ & $u_{m 2}$ & $\cdots$ & $u_{m n}$ \\
\hline
\end{tabular}

Table 2. The expected utility of investment.

\begin{tabular}{|c|c|c|c|c|}
\hline State\&probability & $d_{1}$ & $d_{2}$ & $d_{3}$ & $d_{4}$ \\
\hline$N_{1}: P\left(N_{1}\right)=0.2$ & $u_{11}=100$ & $u_{12}=70$ & $u_{13}=60$ & $u_{14}=-80$ \\
\hline$N_{2}: P\left(N_{2}\right)=0.4$ & $u_{21}=70$ & $u_{22}=80$ & $u_{23}=70$ & $u_{24}=-60$ \\
\hline$N_{3}: P\left(N_{3}\right)=0.2$ & $u_{31}=50$ & $u_{32}=60$ & $u_{33}=80$ & $u_{34}=-40$ \\
\hline$N_{4}: P\left(N_{4}\right)=0.15$ & $u_{41}=-20$ & $u_{42}=10$ & $u_{43}=30$ & $u_{44}=-20$ \\
\hline$N_{5}: P\left(N_{5}\right)=0.05$ & $u_{51}=-100$ & $u_{52}=-80$ & $u_{53}=-40$ & $u_{54}=0$ \\
\hline
\end{tabular}

Table 3. The likelihood ratio.

\begin{tabular}{|c|c|c|c|c|c|}
\hline Likelihood ratio $P\left(X_{j} \mid N_{i}\right)$ & $X_{1}$ & $X_{2}$ & $X_{3}$ & $X_{4}$ & $X_{5}$ \\
\hline$N_{1}: P\left(N_{1}\right)=0.2$ & 0.5 & 0.2 & 0.15 & 0.1 & 0.05 \\
\hline$N_{2}: P\left(N_{2}\right)=0.4$ & 0.2 & 0.5 & 0.2 & 0.05 & 0.05 \\
\hline$N_{3}: P\left(N_{3}\right)=0.2$ & 0.1 & 0.2 & 0.5 & 0.15 & 0.05 \\
\hline$N_{4}: P\left(N_{4}\right)=0.15$ & 0.05 & 0.15 & 0.2 & 0.5 & 0.1 \\
\hline$N_{5}: P\left(N_{5}\right)=0.05$ & 0.05 & 0.1 & 0.15 & 0.2 & 0.5 \\
\hline
\end{tabular}


Table 4 . The posterior probability.

\begin{tabular}{|c|c|c|c|c|c|}
\hline Posterior probability $P\left(N_{i} \mid X_{j}\right)$ & $N_{1}$ & $N_{2}$ & $N_{3}$ & $N_{4}$ & $N_{5}$ \\
\hline$X_{1}$ & 0.4762 & 0.3810 & 0.0952 & 0.0357 & 0.0119 \\
\hline$X_{2}$ & 0.1301 & 0.6504 & 0.1301 & 0.0732 & 0.0163 \\
\hline$X_{3}$ & 0.1212 & 0.3232 & 0.4040 & 0.1212 & 0.0303 \\
\hline$X_{4}$ & 0.1290 & 0.1290 & 0.1935 & 0.4839 & 0.0645 \\
\hline$X_{5}$ & 0.1250 & 0.2500 & 0.1250 & 0.1875 & 0.3125 \\
\hline
\end{tabular}

Table 5. The posterior expected utility

\begin{tabular}{|c|c|c|c|c|}
\hline Posterior expected utility $E\left(d_{j} \mid X_{k}\right)$ & \multicolumn{1}{|c|}{$d_{1}$} & \multicolumn{1}{c|}{$d_{2}$} & \multicolumn{1}{c|}{$d_{3}$} & \multicolumn{1}{c|}{$d_{4}$} \\
\hline$X_{1}$ & 77.14 & 68.93 & 63.45 & -65.48 \\
\hline$X_{2}$ & 61.95 & 68.37 & 65.28 & -56.10 \\
\hline$X_{3}$ & 49.49 & 57.37 & 64.65 & -47.68 \\
\hline$X_{4}$ & 15.48 & 30.65 & 44.19 & -35.48 \\
\hline$X_{5}$ & 1.25 & 13.13 & 28.13 & -33.75 \\
\hline
\end{tabular}

